\title{
Resultados preliminares de um sistema computadorizado e estereoscópico para pupilometria in vivo
}

\author{
Preliminary results of a computerized and stereoscopic system for in vivo \\ pupillometry
}

\author{
Luis Alberto Vieira de Carvalho ${ }^{1}$ \\ AugustoParanhos Júnior ${ }^{2}$
}

Trabalho realizado no Instituto de Física de São Carlos da Universidade de São Paulo - USP - São Carlos (SP) - Brasil e Instituto Israelita de Ensino e Pesquisa Albert Einstein - IIEPAE - São Paulo (SP) - Brasil.

${ }^{1}$ Doutor, Professor de Pós-graduação do Departamento de Oftalmologia da Universidade Federal de São Paulo - UNIFESP - São Paulo (SP) - Brasil; Professor visitante do Instituto de Física de São Carlos da Universidade de São Paulo - USP - São Carlos (SP) - Brasil.

${ }^{2}$ Doutor, Professor afiliado do Departamento de Oftalmologia da UNIFESP - São Paulo (SP) - Brasil; coordenador de Pesquisa Clínica do Instituto Israelita de Ensino e Pesquisa Albert Einstein - IIEPAE - São Paulo(SP) - Brasil.

Endereço para correspondência: Luis Alberto Vieira de Carvalho. Rua Trabalhador São Carlense, 400 - São Carlos (SP) CEP 13560-970

E-mail: lavcf@if.sc.usp.br

Recebido para publicação em 21.12.2006

Última versão recebida em 29.09.2008

Aprovação em 29.09.2008

Nota Editorial: Depois de concluída a análise do artigo sob sigilo editorial e com a anuência da $\mathrm{Dr}^{\mathrm{a}}$ Maria Regina Catai Chalita e do Dr. Fábio Henrique Cacho Casanova sobre a divulgação de seus nomes como revisores, agradecemos suas participações neste processo.

\section{RESUMO}

Objetivos: Desenvolver um sistema digital, com capacidade de induzir acomodação, para medida do diâmetro e formato da pupila para dez diferentes níveis de iluminação (LUX), variando do nível mesópico ao fotópico. Métodos: Um sistema óptico para conjugar imagens da pupila iluminada por LED infravermelho e branco foi projetado e montado usando um capacete típico de oftalmoscópio e uma máscara típica de mergulhador como suporte e uma câmera CCD de alta resolução e sensibilidade. Algoritmos usando princípios de processamento de imagens foram implementados para leitura do arquivo de vídeo e a detecção automática do centro geométrico e diâmetro da pupila para cada quadro. Resultados: Apresentamos dados preliminares deste sistema para um paciente voluntário. Os dados sobre posição horizontal (x), vertical (y) do centro geométrico e do diâmetro da pupila foram exportados para arquivos texto que podem ser impressos usando um programa convencional de planilha (Excel). O processo de acomodação é garantido por uma mira virtual (LED branco) localizada a 6 metros do olho do paciente e o nível de iluminaçãoé precisamente controlado por uma placa eletrônica que alimenta o LED central que tem sua potência controlada pelo "software". Conclusões: Com o instrumento desenvolvido dados precisos da posição e tamanhos da pupila podem então ser obtidos estereoscopicamente (para ambas as pupilas e ao mesmo tempo) para qualquer paciente. Acreditamos que tal instrumento possa ser útil em algumas práticas oftálmicas onde as características geométricas da pupila sejam importantes.

Descritores: Pupilometria/métodos; Procedimentos cirúrgicos refrativos/métodos; Pupila/fisiologia; Tecnologia de equipamentos e provisões; Algoritmos; Sensibilidade de contraste; Estimulação luminosa

\section{INTRODUÇÃO}

Alguns textos dão à pupila unicamente a função de controle de quantidade de luz que adentra o olho, de maneira análoga ao diafragma de uma máquina fotográfica ${ }^{(1-2)}$. No entanto, ao contrário do que se pensa, sua função não é apenas controlar a luminosidade que incide na retina, mas também regular a profundidade de foco ${ }^{(3)}$. Para focalizar objetos distantes o seu diâmetro diminui, e para objetos próximos o diâmetro aumenta, de maneira involuntária, mas altamente precisa ${ }^{(4)}$, daí a importância de se estabilizar não somente a quantidade de iluminação, mas também a acomodação do cristalino para se determinar o diâmetro pupilar. Não coincidentemente o diafragma de uma boa máquina fotográfica manual também pode ser usado para alterar a profundidade focal, algo que todo "bom” fotógrafo está familiarizado. 
Além dos fatores relacionados à qualidade visual, existem outras situações para as quais a pupila realiza movimentos em sua amplitude, como: estado emocional, nível de atenção, entre outros. Neste trabalho não estudamos estes fatores; estudamos apenas aqueles relacionados à intensidade luminosa para um olho fixando no infinito (> $6 \mathrm{~m})$.

Outro motivo importante para caracterização dos parâmetros pupilares são as modernas cirurgias refrativas a laser ${ }^{(5-7)}$. Estas cirurgias consistem na aplicação de luz laser em diferentes posições da córnea com o objetivo de retirar camadas de células e "moldar" a córnea no formato que otimize o processo de refração e permita formação de imagens mais nítidas na fóvea para correção de miopia, astigmatismo e hipermetropia. $\mathrm{Na}$ figura 1 ilustramos a vista superior da região operada para o mesmo olho com três tamanhos e posições diferentes da pupila.

Na figura 1 temos três casos hipotéticos para um mesmo olho: (A) pupila para nível fotópico de luz (> 4 LUX). Na iluminação fotópica a pupila adquire forma com diâmetros bem pequenos ( $2 \mathrm{~mm})$ e, portanto é bem menor que a região operada pelo laser (normalmente de $6 \mathrm{~mm}$ ). Para este caso a visão será boa mesmo que o centro da região operada não coincida exatamente com o centro da pupila, pois aqui somente raios paraxiais estão entrando no olho e a imagem na retina conseqüentemente só depende do poder óptico da região central da córnea; (B) para níveis de iluminação escotópica e mesópica $(<4$ LUX) os diâmetros pupilares podem chegar a $8 \mathrm{~mm}$, portanto além da região operada. Se, para estes casos a cirurgia for descentrada com relação à córnea e o centro da pupila não coincidir com o centro da córnea, regiões de entrada de luz não corrigidas podem influenciar na visão noturna do paciente (por exemplo, quando estiver dirigindo à noite); (C) mesmo para centros que coincidam, a informação do diâmetro pupilar pode indicar ao médico tam- bém a necessidade em aumentar a região cirúrgica, quando possível. A maioria dos lasers disponíveis hoje comercialmente não permite a mudança da área de cirurgia, pois isto é calculado automaticamente pelo "software", mas acreditamos que no futuro isso será possível, pois é mais um parâmetro para otimizar a cirurgia personalizada.

Portanto, um sistema que possa quantificar com precisão a posição do centro e o diâmetro pupilar para diferentes níveis de luz e para sequiências de vídeo e não somente para imagens estáticas, é algo que vem de encontro às necessidades da comunidade oftálmica. É especialmente útil para aqueles médicos que realizam cirurgia refrativa a laser com instrumentos que possam levar em conta informações personalizadas de cada paciente (nas chamadas cirurgias personalizadas ${ }^{(5)}$ ).

\section{MÉTODOS}

\section{Instrumentação}

Para realizar vídeos digitais da pupila adaptamos um sistema óptico no capacete de um oftalmoscópio desenvolvido pela empresa Eyetec Equipamentos Oftálmicos (http:// www.eyetec.com.br/ohd.php). Desta maneira o paciente, ao invés de olhar através de um sistema fixo de bancada, pode então movimentar a cabeça e ficar em posição ereta, dando maior conforto para exames mais longos. O sistema todo, já montado, pode ser visto na figura 2 .

No sistema óptico, além das lentes de aumento, há um LED infravermelho e um LED visível para iluminação da pupila e a intensidade destes LEDs pode ser controlada externamente. Há também no sistema óptico um LED visível que funciona como objeto de uma lente que projeta sua imagem em um plano virtual localizada há 6 metros do olho do paciente. Desta maneira o paciente pode fixar o olhar durante o exame e manter

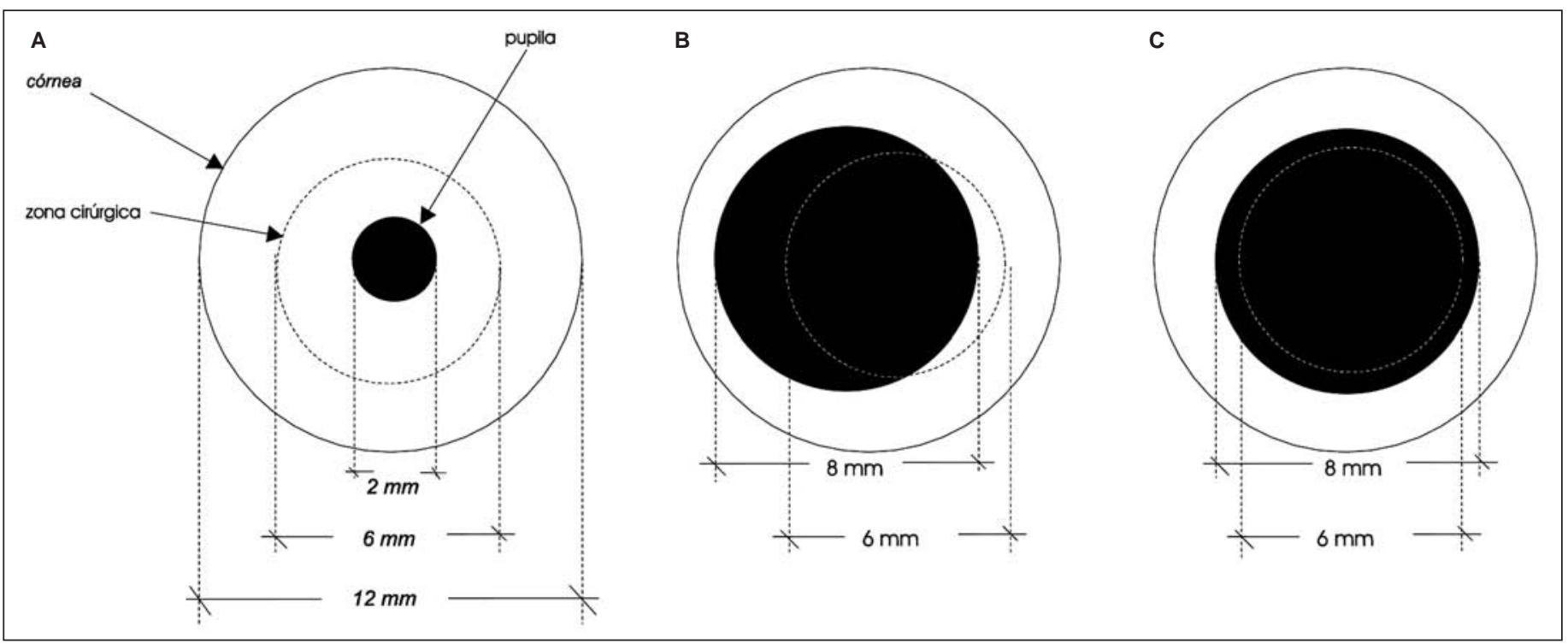

Figura 1 - Diferentes tamanhos e posições de pupila podem e devem definir diferentes procedimentos cirúrgicos 


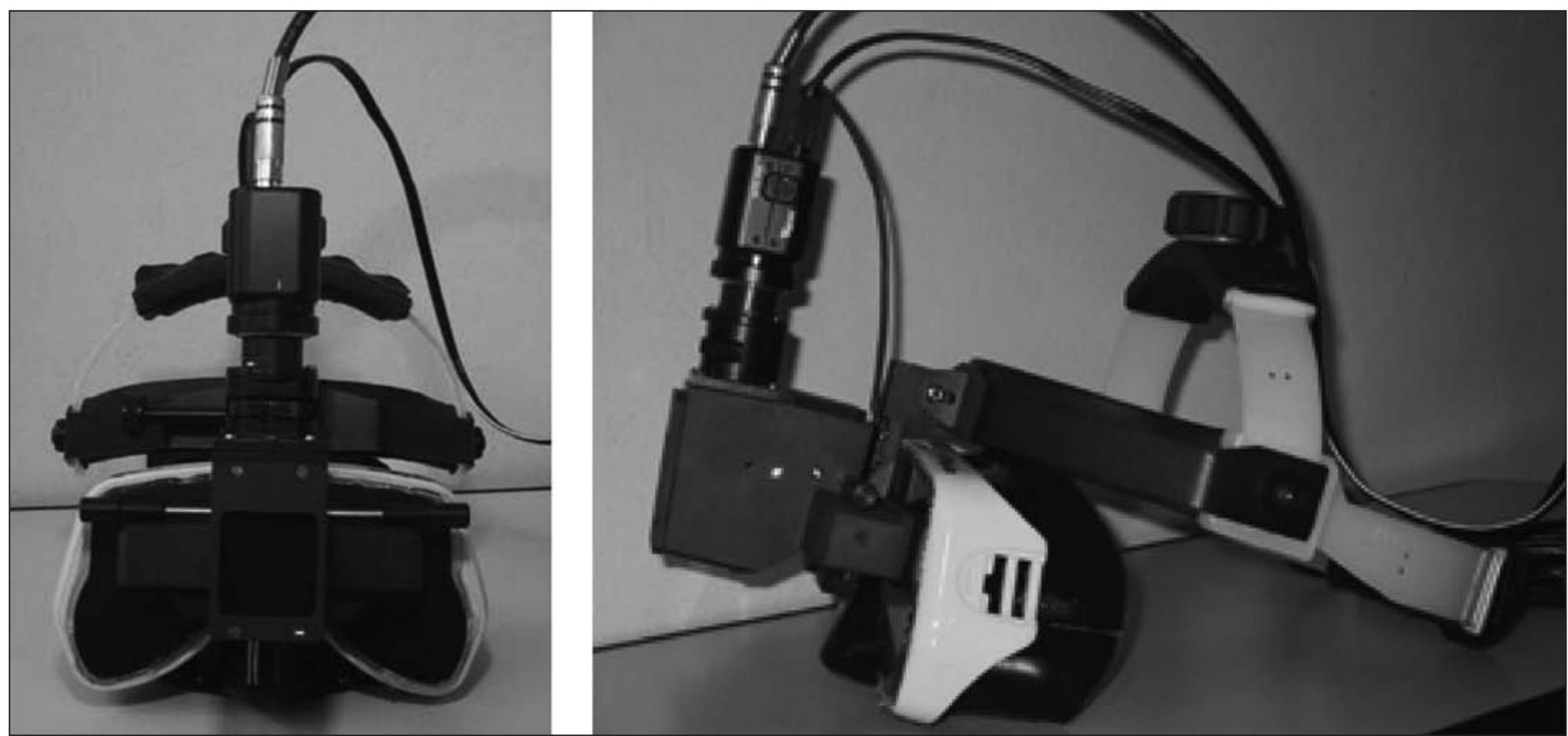

Figura 2 - Fotos frontal (esquerda) e lateral (direita) do novo pupilômetro desenvolvido no laboratório de P\&D da Eyetec Equipamentos Oftálmicos

o cristalino acomodado para o infinito (na realidade, desacomodado). A parte eletrônica controla a intensidade de luz usando a porta serial do PC onde podemos também digitalizar imagens da pupila bastante nítidas. A captura das imagens é realizada por uma placa "frame grabber" comercial instalada em um PC (Pentium III, $800 \mathrm{MHz}$ ). A parte do "software" que controla a intensidade de luz foi desenvolvida em Pascal (Delphi) (www.borland.com) e a parte que realiza o processamento dos vídeos foi desenvolvida em Matlab (www.mathworks.com). Pela interface gráfica do "software" o usuário pode escolher até dez níveis diferentes de iluminação (de mesópico a fotópico) e determinar os intervalos entre cada mudança de intensidade de luz. Portanto, é o usuário que determina o tempo total de exposição e duração do exame. A máscara evita que a luz vinda do ambiente influencie no processo do exame. Na figura 3, mostramos um dos quadros de uma seqüência de vídeo sem magnificação que foi digitalizado para um voluntário (sexo masculino, 31 anos).

\section{"Software" para processamento}

Para desenvolvimento do algoritmo para detecção dos parâmetros da pupila utilizamos técnicas convencionais em processamento de imagens ${ }^{(8)}$ e alguns princípios de varredura em coordenadas polares utilizados em trabalhos anteriores para processamento de imagens dos Discos de Plácido $^{(9-14)}$. A figura 4 ilustra o princípio utilizado para encontrar os parâmetros pupilares desejados.

$\mathrm{O}$ algoritmo inicia a busca pelo centro da imagem $\left(x_{\mathrm{cg} 1}, y_{\mathrm{cg} 1}\right)$ e percorre em ambas as direções (vertical e horizontal) em ambos sentidos até encontrar pontos de borda $\left(\mathrm{P}_{1} \ldots \mathrm{P}_{4}\right)$. A mediatriz de $P_{1} P_{2}$ e $\mathrm{P}_{3} \mathrm{P}_{4}$ fornece as coordenadas do novo ponto

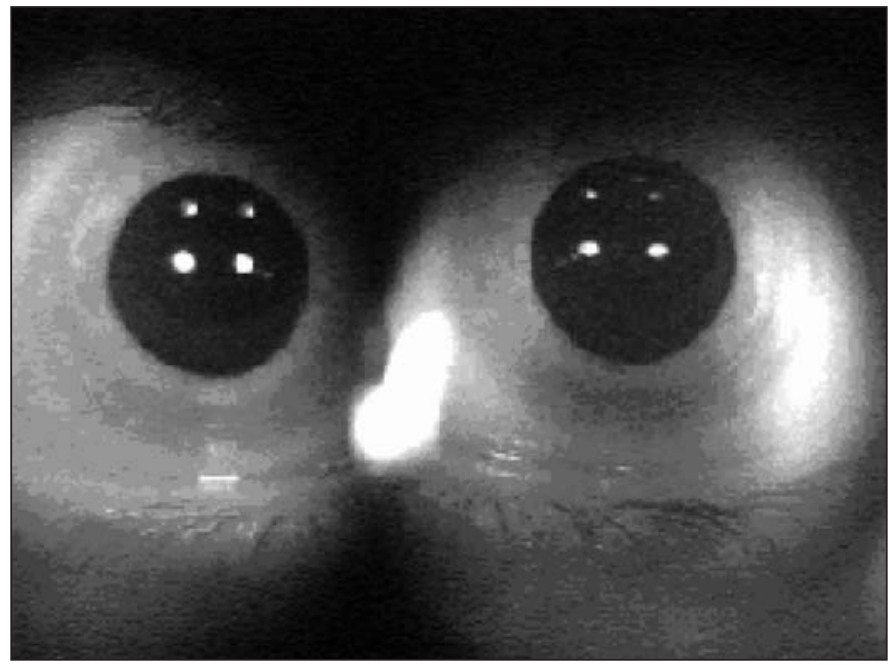

Figura 3 - Imagem típica do novo pupilômetro digital. Para toda a seqüência de vídeo o movimento de ambas as pupilas é gravado simultaneamente de tal maneira que se pode obter dados estereoscópicos do sistema. A imagem acima é apenas ilustrativa e, portanto não está filtrada com todos os algoritmos que fazem parte do processamento das bordas da pupila para um ser humano é difícil identificar as bordas nestas imagens

originais, mas para o "software" é uma tarefa bem mais precisa.

de busca $\left(x_{\mathrm{cg} 2}, y_{\mathrm{cg} 2}\right)$. A partir deste novo ponto o algoritmo inicia a busca pelos vários pontos que caracterizam a borda da pupila, ou seja, a região de transição entre o diafragma da íris (que é a pupila) e a parte colorida da íris. Esta busca é realizada em coordenadas polares, para o ângulo azimutal $(\theta)$ variando de 1 a $360^{\circ}$, em intervalos de $1^{\circ}$ e para a distância radial $(\rho)$ variando de zero até o ponto de transição pupila-íris. O centro geométrico da pupila é então determinado como sendo, 


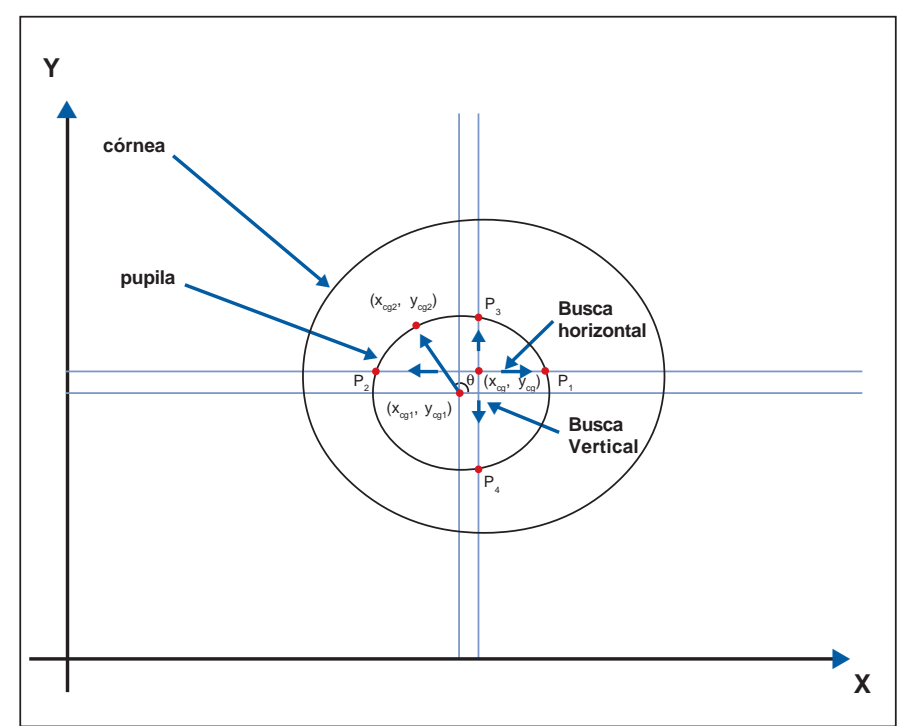

Figura 4 - Parâmetros pupilares e estratégia para processamento

$$
\begin{aligned}
& X_{c m}=\sum_{i=1}^{360} x_{b}^{i} \\
& Y_{c m}=\sum_{i=1}^{360} y_{b}^{i}
\end{aligned}
$$

onde $\left(x_{\mathrm{b}}, y_{\mathrm{b}}\right)$ são as coordenadas dos pontos de borda. Depois de determinado o centro geométrico (ou centro de "massa") determinamos o raio médio da circunferência que melhor aproxima os pontos de borda calculando a média de 360 distâncias

$$
d_{i}=\sqrt{\left(x_{b}^{i}-x_{c m}\right)^{2}+\left(y_{b}^{i}-y_{c m}\right)^{2}}
$$

onde

$$
d_{m}=\sum_{i=1}^{360} d_{i}
$$

é a distância média (ou raio da circunferência aproximada) em mm. O diâmetro médio é simplesmente a equação (4) multiplicado por dois. A seguir mostramos alguns resultados práticos do sistema desenvolvido.

\section{RESULTADOS}

Como mencionado anteriormente, realizamos apenas medidas preliminares neste trabalho e apresentamos aqui, por motivos didáticos, apenas os resultados para um dos olhos, embora o instrumento possa ser utilizado para medidas estereoscópicas. Uma seqüência de 30 segundos de vídeo foi capturada para um paciente voluntário (sexo masculino, 31 anos) com intensidade de iluminação variando de 0 a 5 LUX. $\mathrm{O}$ arquivo de vídeo foi então inserido em nosso "software" e para todos os quadros foram determinados o centro geométrico (coordenadas X e Y) e diâmetro da pupila. Na figura 5 mostramos uma das imagens do voluntário sendo processada pelo "software" e na figura 6 mostramos um gráfico com a seqüência de dados.

Como podemos ver pela figura 7 os movimentos da pupila na direção vertical (Y) variaram entre aproximadamente 750 e 1050 microns sendo que na direção horizontal (X) a variação foi aproximadamente entre -50 e 100 microns. O gráfico mostra esta variação de maneira mais clara, pois nele podemos ver todas as posições do centro da pupila ao longo de todo o processo de medida.

Os dados para o diâmetro médio para cada um dos quadros para esta mesma seqüência pode ser visto na figura 8 .

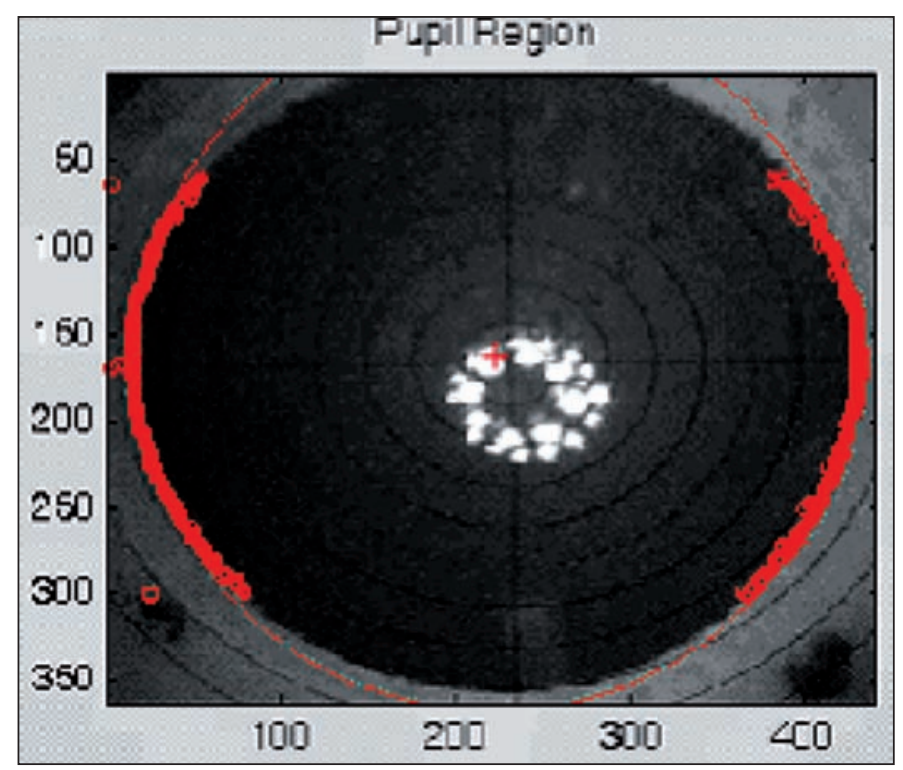

Figura 5 - Tela do programa no momento em que estava encontrando a borda da pupila para um dos quadros da seqüência de imagens de vídeo

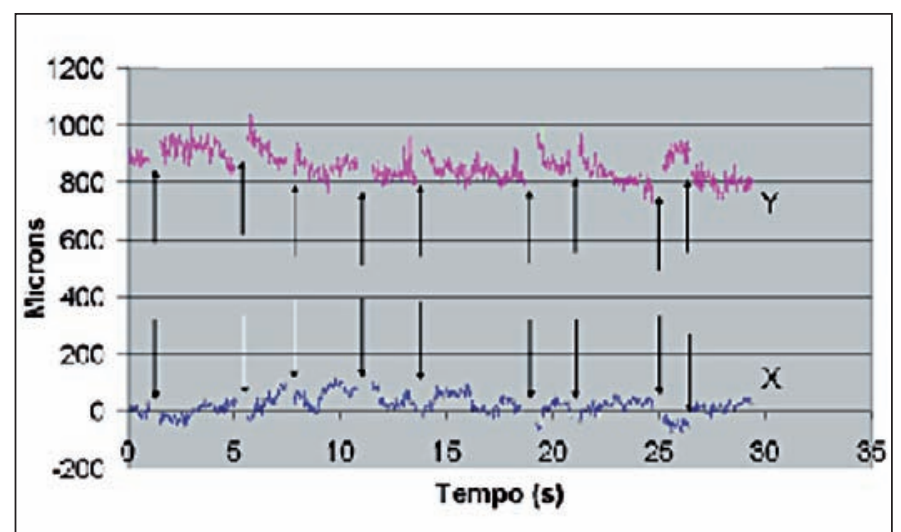

Figura 6 - Movimento em $\mathrm{X}$ e $\mathrm{Y}$ do centro da pupila para uma seqüência de aproximadamente meio minuto (30 segundos) para o paciente voluntário. Como podemos verificar o centro geométrico da pupila é precisamente monitorado em ambas as direções. 


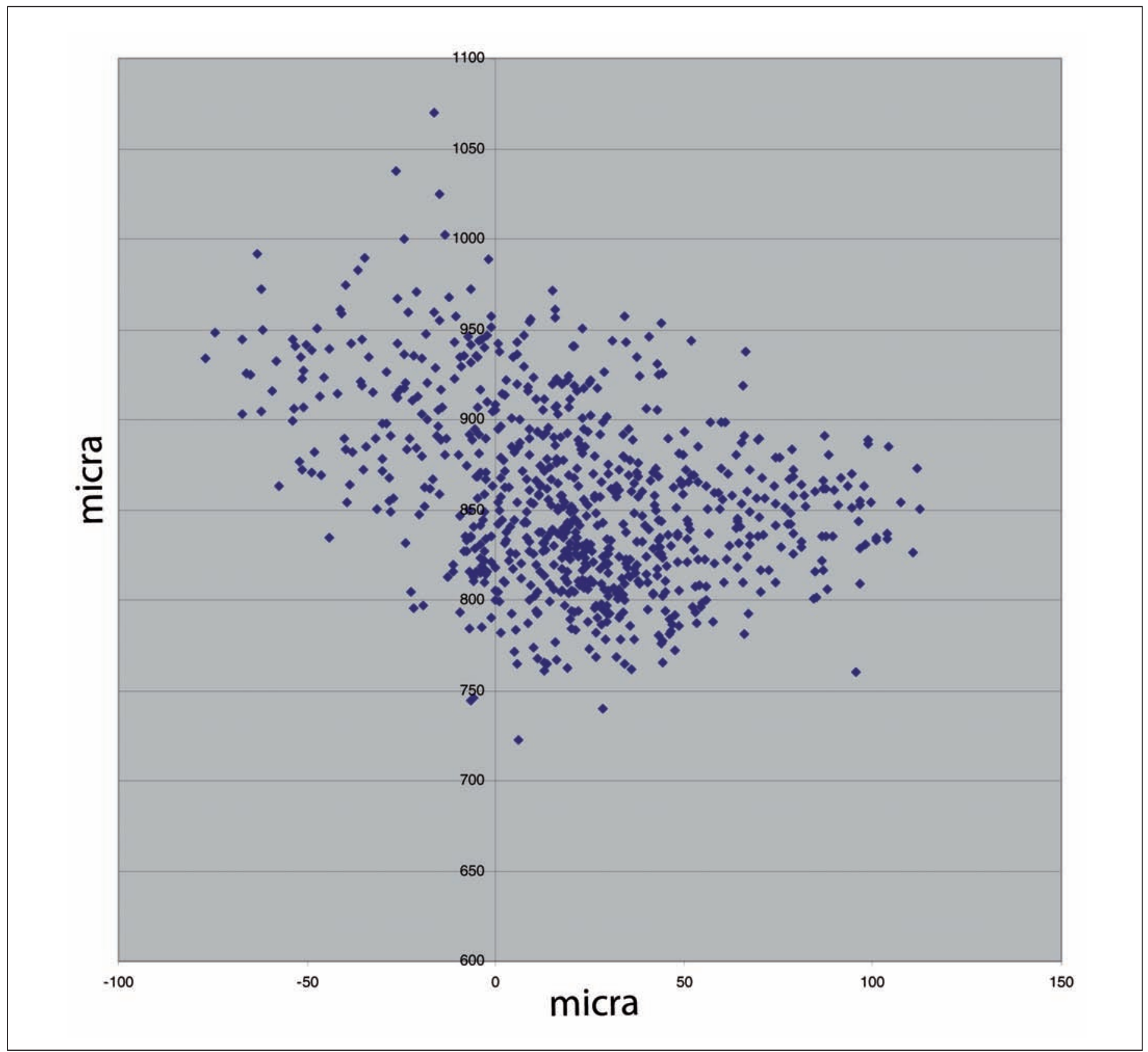

Figura 7 - Posições do centro da pupila para toda a seqüência de vídeo mostrada na figura anterior

\section{DISCUSS ÃO}

Apresentamos o "hardware" e "software" de um instrumento preciso para medidas da pupila (pupilômetro) in vivo para condições luminosas que simulam as condições do dia-a-dia (noturno e diurno). Os resultados são promissores, pois possibilitam a medida em tempo real para ambos os olhos (estereoscópico) e apresentam coerência com as diferentes intensidades de luz, ou seja, diminuição do diâmetro pupilar com o aumento da luminosidade para acomodação no infinito. Além disso, pelo fato de possibilitar medidas estereoscópicas, permite não somente que medidas em função da acomodação e luminosidade sejam realizadas, mas também como as variações da pupila em função da vergência, algo impossível num instrumento monocular ${ }^{(4)}$. Como a variação pupilar estereoscópica é diferente da monocular para quaisquer condições de acomodação, este instrumento também pode ser usado para melhor previsão da área cirúrgica ideal para ambos os olhos em casos de cirurgia refrativa.

Apesar dos bons resultados apresentados aqui para o nosso paciente voluntário, faz-se necessário algumas observações para casos especiais. A pupila mensurada nestes testes preliminares era bastante simétrica (formato bastante pró- 


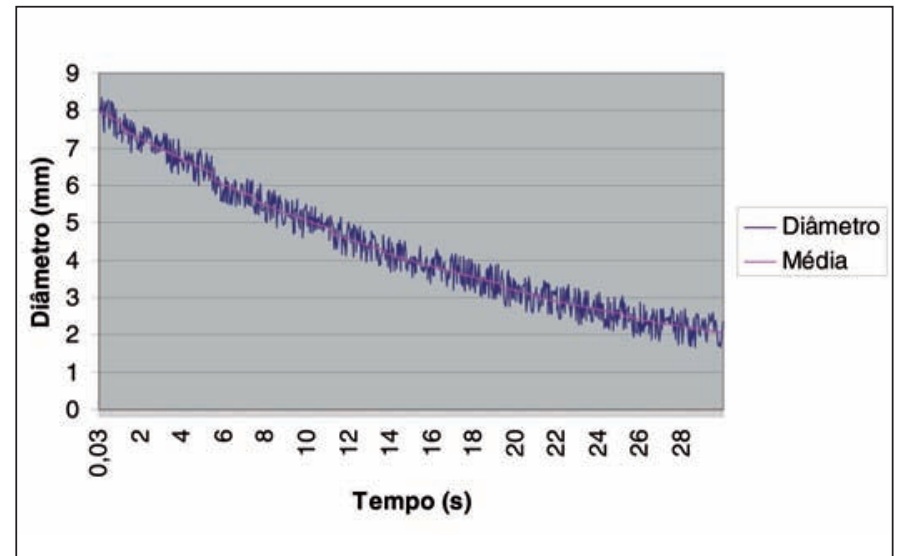

Figura 8 - Variação do diâmetro da pupila do olho direito para o paciente voluntário para toda a seqüência de vídeo (aproximadamente $30 \mathrm{~s}, 765$ quadros), com variação controlada de luz de 0 a 5 LUX, em passos de 0,5 LUX

ximo a um círculo perfeito), portanto o método dos mínimos quadrados usando a equação de uma circunferência (veja equação 3) funcionou bastante bem. No entanto, para pacientes com pupilas não simétricas (alteração de forma), pupilas patológicas, pós-operados com corectopia ou discoria, o software deste instrumento deve ser aprimorado. Como o princípio utilizado aqui calcula o melhor raio de curvatura como uma média de todos os raios para 360 graus, o sistema sempre vai partir da hipótese de que a pupila do paciente é aproximadamente circular. Para casos em que isso não é verdade a aproximação pela melhor circunferência pode induzir em aproximações não otimizadas opticamente para o centro de visão e para o diâmetro total da região a ser ablada, em casos pré-cirurgia refrativa. Em breve pretendemos adicionar ao software do instrumento a possibildade de calcular o melhor centro de "massa" da pupila e contornos não simétricos.

De modo geral, acreditamos que o software e hardware apresentados aqui para este instrumento, após algumas melhorias, possam servir ao médico como mais uma técnica no diagnóstico de patologias do olho e também no planejamento personalizado de cirurgias refrativas, ou em outros casos onde seja necessário o conhecimento preciso do diâmetro e posição da pupila para condições adversas de luminosidade. Em futuros estudos pretendemos analisar tal instrumento clinicamente para uma população estatisticamente significativa.

\section{ABSTRACT}

Purpose: We developed a digital system with accommodation capacities for measurement of the diameter and shape of the in vivo pupil for 10 different levels of illumination intensity, varying from mesopic to photopic. Methods: An optical system for conjugating images from an infrared and white light illuminated pupil was designed and mounted using an ophthalmoscope helmet and a typical diving mask as support for a high-resolution and sensitivity CCD. Using an IBM compatible computer sequences of video in AVI format were digitized for several seconds at a mean rate of $30 \mathrm{~Hz}$. Algorithms using principles of image processing were implemented for detection of the pupil edges. Results: We present preliminary results of this system for a voluntary patient. Data for the horizontal (x) and vertical (y) central position and for the diameter of the pupil were then exported to files that could be read by typical spread sheet programs (Excel). Conclusions: In this manner, precise data can be obtained stereoscopically (for both pupils at the same time) for any patient, given that the accommodation process is guaranteed by using a white LED virtual mire located 6 meters from the patient's eye. An electronic board precisely controls the level of illumination. We believe here developed instrument may be useful in certain ophthalmic practices where precise pupil geometric data are needed.

Keywords: Pupilometry/methods; Refractive surgical procedures/methods; Pupil/physiology; Equipment and supplies technology; Algorithms; Contrast sensitivity; Photic stimulation

\section{REFERÊNCIAS}

1. Le Grand Y. Physiological optics. Berlin: Springer-Verlag; 1980. [Springer series in optical sciences: v.13].

2. Okuno E, Caldas IL, Chow C. Física para ciências biológicas e biomédicas. São Paulo: Harper \& Row do Brasil; 1982.

3. Förster S, Gross H, Höller, Höring L. Extended depth of focus as a process of pupil manipulation [Internet]. Proceedings of SPIE. 2005. v.5962 [cited 2008 Oct 24). Available from: https://scitation.aip.org/journals/doc/SPIEDLhome/spieLogin.jsp. DOI:10.1117/12.624814.

4. Rosen ES, Gore CL, Taylor D, Chitkara D, Howes F, Kowalewski E. Use of a digital infrared pupillometer to assess patient suitability for refractive surgery. J Cataract Refract Surg. 2002;28(8):1433-8.

5. Krueger RR. Technology requirements for Summit-Autonomous CustomCornea. J Refract Surg. 2000;16(5):S592-601.

6. Campin JA, Pettit GH, Gray GP. Required laser beam resolution and PRK system configuration for custom high fidelity corneal shaping. Invest Ophthalmol Vis Sci. 1999;38(Suppl): S538.

7. Carvalho LAV, Castro JC, Schor P, Chamon W, Carvalho L. Wave-front measurements of the human eye using the Hartmann-Shack sensor and current state-of-the-art technology for excimer laser refractive surgery. Lasik and Beyond Lasik, Section V, Chapter 31, Highlights of Ophthalmology, 2001.

8. Gonzales RC, Woods RE. Digital image processing. Reading, Mass.: Addison-Wesley; c1992. p.190.

9. Carvalho LA, Stefani M, Romão AC, Tonissi S, Castro JC. Processamento digital de imagens refletidas pelo filme lacrimal da superfície anterior da córnea. Rev Bras Eng Biom = Braz J Biomed Eng. 2001;17(3):113-22.

10. Carvalho LA, de Faria e Souza SJ, Castro JC. Desenvolvimento de um sistema para medida da curvatura da córnea durante o ato cirúrgico. Rev Fis Apl Instrum. 1996;11(3):119-26.

11. Carvalho LA, Paranaguá E, Santos LE, Tonissi SA, Romão AC, Oliveira $\mathrm{AC}$, et al. Desenvolvimento de um instrumento computadorizado para avaliação da curvatura da córnea humana. Rev Fis Apl Instrum. 1997;12(3): 81-90.

12. Carvalho LAV, Tonissi SA, Romão AC, Santos LE, Yasuoka F, Oliveira AC, et al. Desenvolvimento de um instrumento computadorizado para medida do poder refrativo da córnea (videoceratógrafo). Arq Bras Oftalmol. 1998;61(6): 640-54.

13. Carvalho L, Tonissi SA, Castro JC. Preliminary tests and construction of a computerized quantitative surgical keratometer. J Cataract Refract Surg. 1999; 25(6):821-6. Comment in: J Cataract Refract Surg. 2000;26(8):1104-5.

14. Plácido A. Novo instrumento de exploração da córnea. d'Oftalmológica Practica (Lisboa). 1880;5:27-30. 